\title{
High-Efficiency Intracavity Continuous-Wave Green-Light Generation by Quasiphase Matching in a Bulk Periodically Poled MgO:LiNbO 3 Crystal
}

\author{
Shaowei Chu, ${ }^{1,2}$ Ying Zhang, ${ }^{3}$ Bin Wang, ${ }^{1}$ and Yong $\mathrm{Bi}^{1}$ \\ ${ }^{1}$ Division of Opto-Electronics System, Academy of Opto-Electronics, Chinese Academy of Sciences, Beijing 100085, China \\ ${ }^{2}$ Graduate University of Chinese Academy of Sciences (GUCAS), Beijing 100080, China \\ ${ }^{3}$ R\&D Department, Phoebus Vision Opto-Electronics Technology Ltd., Beijing 100094, China
}

Correspondence should be addressed to Yong Bi, biyong@aoe.ac.cn

Received 29 March 2008; Accepted 18 August 2008

Recommended by Yalin Lu

$908 \mathrm{~mW}$ of green light at $532 \mathrm{~nm}$ were generated by intracavity quasiphase matching in a bulk periodically poled $\mathrm{MgO}_{\mathrm{LiNbO}}$ (PPMgLN) crystal. A maximum optical-to-optical conversion efficiency of $33.5 \%$ was obtained from a $0.5 \mathrm{~mm}$ thick, $10 \mathrm{~mm}$ long, and $5 \mathrm{~mol} \% \mathrm{MgO}: \mathrm{LiNbO}_{3}$ crystal with an end-pump power of $2.7 \mathrm{~W}$ at $808 \mathrm{~nm}$. The temperature bandwidth between the intracavity and single-pass frequency doubling was found to be different for the PPMgLN. Reliability and stability of the green laser were evaluated. It was found that for continuous operation of 100 hours, the output stability was better than $97.5 \%$ and no optical damage was observed.

Copyright (c) 2008 Shaowei Chu et al. This is an open access article distributed under the Creative Commons Attribution License, which permits unrestricted use, distribution, and reproduction in any medium, provided the original work is properly cited.

\section{Introduction}

Compact and efficient green laser light sources have numerous applications such as laser displays, material processing, biological investigations, and optical communications. There are many methods to achieve coherent green light; however, second harmonic generation (SHG) by the quasi-phasematching (QPM) technique has been an attractive method to obtain compact and high-efficiency laser [1]. The QPM technique based on periodically poled lithium niobate (PPLN) has significant advantages including phase matching of an arbitrary wavelength by the use of an appropriate period of polarization inversion and a higher nonlinear coefficient than KTP, LBO, and BIBO.

So far, the single-pass SHG scheme is a popular solution for achieving CW green laser light. However, this scheme requires a high nonlinear coefficient and a long interaction length to achieve high conversion efficiency, which can be satisfied by employing PPLN crystals. CW green power of $2.7 \mathrm{~W}$ has been obtained in a $50 \mathrm{~mm}$ long PPLN single-pass crystal pumped by a $6.5 \mathrm{~W}$ Nd:YAG laser [2]. Due to its higher photorefractive damage threshold and lower green- induced infrared absorption as compared with PPLN [3], periodically poled $\mathrm{MgO}: \mathrm{LiNbO}_{3}(\mathrm{PPMgLN})$ has replaced the PPLN. A maximum power of $1.18 \mathrm{~W}$ at $531 \mathrm{~nm}$ with $16.8 \%$ conversion efficiency has been obtained from a $2 \mathrm{~mm}$ thick, $25 \mathrm{~mm}$ long PPMgLN single-pass crystal pumped by a $7 \mathrm{~W}$ $\mathrm{Nd}: \mathrm{GdVO}_{4}$ laser [4]. Periodically poled Mg-doped stoichiometric lithium tantalate (PPMgSLT) is usually used as an alternative material for high-power generation. $7 \mathrm{~W}$ of SHG green light with $35.4 \%$ conversion efficiency in a $2 \mathrm{~cm}$ long PPMgSLT single-pass crystal pumped by a $19.6 \mathrm{~W}, 1084 \mathrm{~nm}$ $\mathrm{Yb}$-doped fiber laser have recently been reported [5].

Intracavity second harmonic generation (ISHG) of Nddoped lasers has always been an attractive method for producing green light [6]. Second harmonic generation in a bulk PPLN crystal was demonstrated using the intracavity scheme for the first time in 1995 [7]. In a later experiment, $53 \mu \mathrm{W}$ of green ISHG at $541 \mathrm{~nm}$ were generated with a pump power of $300 \mathrm{~mW}$, indicating $0.02 \%$ optical-to-optical conversion efficiency in 1997 [8]. A maximum output power of $740 \mathrm{~mW}$ of blue light has also been generated with an optical-to-optical efficiency of $5.7 \%$ at a pump power of $13.5 \mathrm{~W}[9]$. 


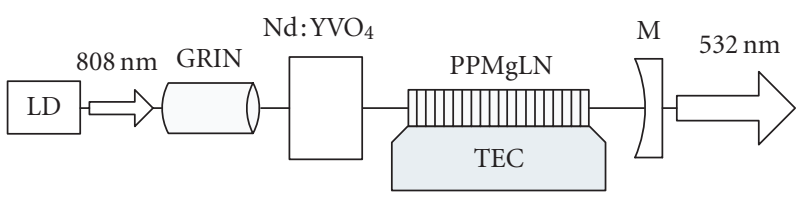

FIGURE 1: Experimental setup used for ISHG.

In this paper, we report highly efficient continuouswave green-light generation based on intracavity frequency doubling, in a quasi-phase-matched PPMgLN bulk crystal. With an end-pump power of $2.7 \mathrm{~W}$ at $808 \mathrm{~nm}$, a maximum green output power of $908 \mathrm{~mW}$ at $532 \mathrm{~nm}$ is achieved with a high optical-to-optical conversion efficiency of $33.5 \%$.

\section{Experiments}

The experimental setup of a high-efficiency CW, laser-diode (LD), and end-pumped green laser with an intracavity SHG scheme is shown schematically in Figure $1 . \mathrm{YVO}_{4}$ doped with $1 \% \mathrm{Nd}$ with a size of $3 \times 3 \times 3 \mathrm{~mm}^{3}$ was used as the gain medium. A $0.5 \mathrm{~mm}$ thick, $10 \mathrm{~mm}$ long, $2 \mathrm{~mm}$ wide, and $5 \mathrm{~mol} \%$ PPMgLN crystal provided by the $\mathrm{C} 2 \mathrm{C}$ Link Corporation, Canada, was used as a frequency doubler. Both sides of the PPMgLN crystal were antireflective (AR), coated at $532 \mathrm{~nm}$ and $1064 \mathrm{~nm}$. Temperature of the PPMgLN was controlled by a thermoelectric cooler (TEC).

The laser cavity consisted of a high-reflection (HR) coating at $1064 \mathrm{~nm}$ and $\mathrm{AR}$ coating at $808 \mathrm{~nm}$ on the pumping side of the Nd:YVO ${ }_{4}$ crystal, as well as a $50 \mathrm{~mm}$ radius of curvature mirror $(\mathrm{M})$ with $\mathrm{HR}$ coating at $1064 \mathrm{~nm}$ and $\mathrm{AR}$ coating at $532 \mathrm{~nm}$, which were used, respectively, for folding the fundamental laser beam and for the second harmonic output. The other side of the $\mathrm{Nd} \mathrm{YVO}_{4}$ crystal was AR-coated at $1064 \mathrm{~nm}$ and HR-coated at $532 \mathrm{~nm}$. The optical end-pump was a CW-2.7 W-laser diode whose endface was imaged into the pump side of the $\mathrm{Nd}: \mathrm{YVO}_{4}$ crystal by a graded index lens (GRIN). The GRIN with a size of $1.8 \times 5(R \times L) \mathrm{mm}^{3}$ was AR-coated at $808 \mathrm{~nm}$.

\section{Experimental Results}

The CW green laser output power versus the pump power is shown in Figure 2. To obtain the data, the $808 \mathrm{~nm}$ pump LD power was varied by changing the injection current of the LD. When the pump power was up to $2.7 \mathrm{~W}$, the green laser delivered $908 \mathrm{~mW}$ with $33.5 \%$ optical-to-optical conversion efficiency at $35.2^{\circ} \mathrm{C}$. To the best of our knowledge, this is the highest optical-to-optical conversion efficiency reported to date for a green laser at low pump power $(<5 \mathrm{~W})$. This conversion efficiency is even higher than that obtained for CW single-pass SHG in a bulk PPMgLN with a $1064 \mathrm{~nm}$ pump power of $7 \mathrm{~W}$ [4]. It is worth noting that the AR coating of the PPMgLN crystal has not been optimized since multiple beam spots were observed in the far-filed pattern of the green laser. We believe that the efficiency can further be enhanced by optimizing the AR coating conditions.

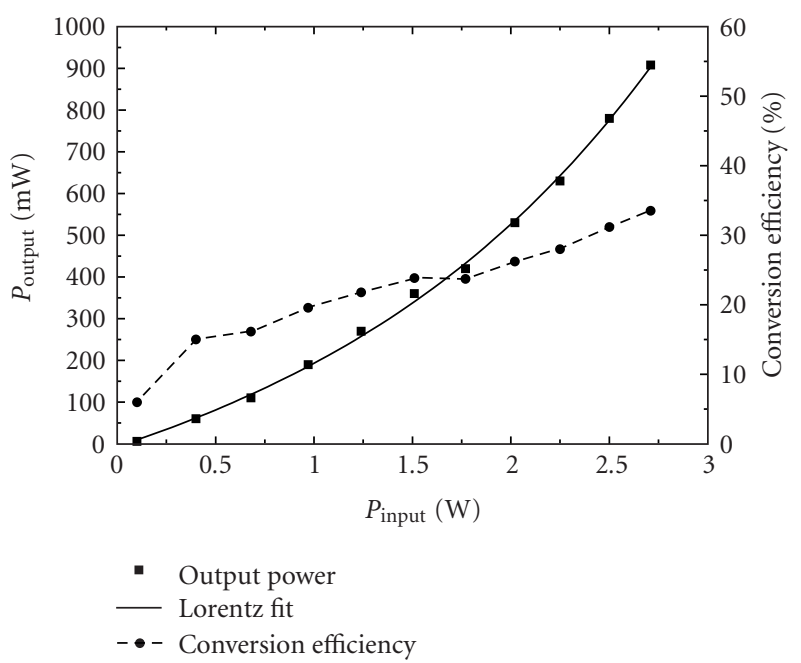

FIGURE 2: $532 \mathrm{~nm}$ green output power and the optical-to-optical conversion efficiency versus $808 \mathrm{~nm}$ input power at phase-matching temperature.

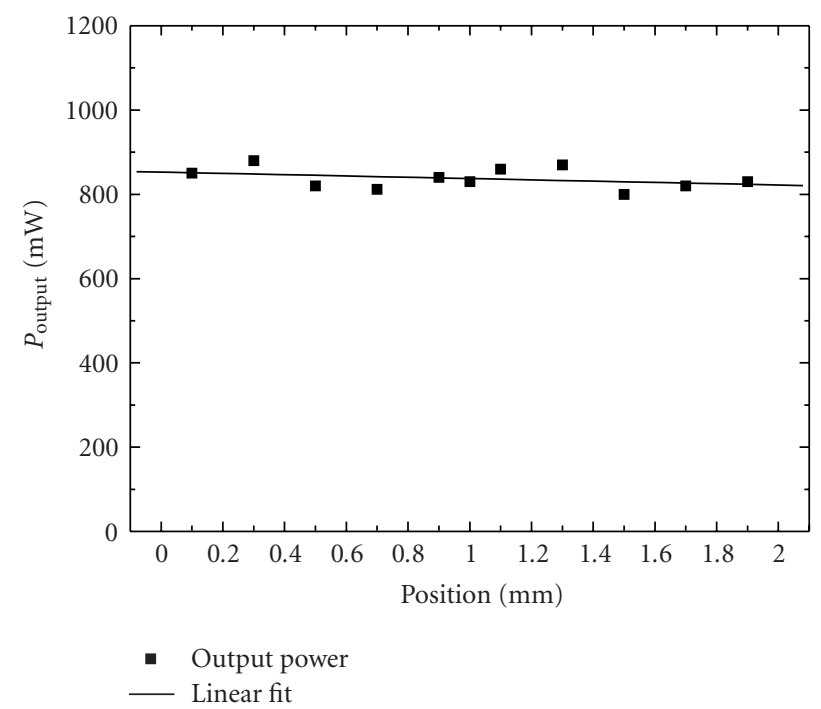

FIGURE 3: $532 \mathrm{~nm}$ output power versus transverse position of PPMgLN crystal at an optical input power of $2.7 \mathrm{~W}$.

In order to measure the uniformity of the PPMgLN crystal at different transverse positions, we shifted the PPMgLN crystal transversely when the temperature was set at the phase-matching temperature, while all other conditions are held constant. As shown in Figure 3, change of the green output power is less than 3\%, indicating high uniformity of the PPMgLN crystal. Therefore, it is possible that several beams can pass a single PPMgLN crystal at the same time, which can further enhance the optical-to-optical conversion efficiency.

Maximum green output power can be obtained at the phase-matching temperature for PPMgLN crystal. In the previous reports, the single-pass phase-matching temperature has been investigated, but the phase-matching temperature of the PPMgLN in a laser cavity has not been 


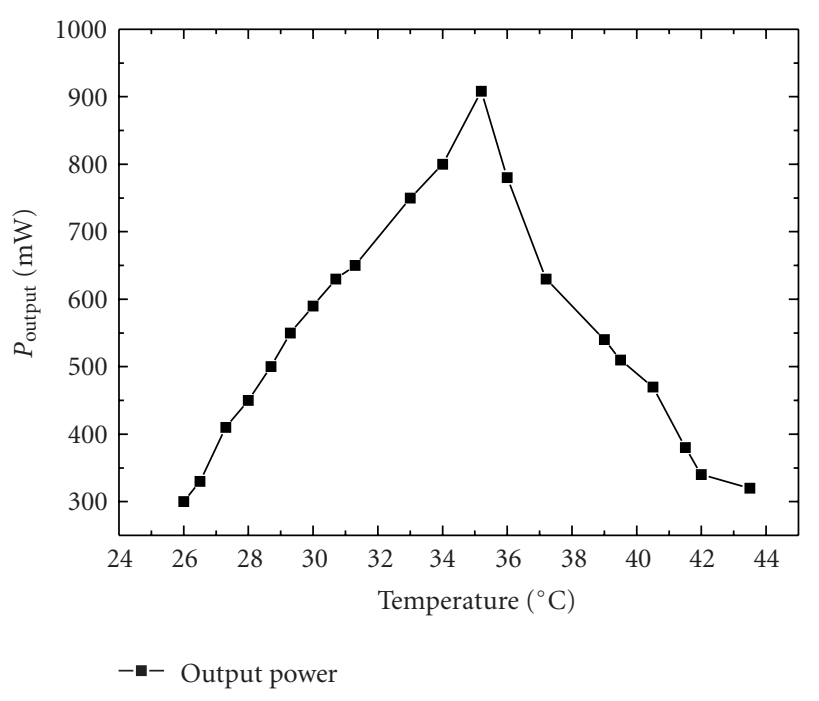

FIGURE 4: $532 \mathrm{~nm}$ output power versus temperature for SHG at an optical input power of $2.7 \mathrm{~W}$.

reported. In this paper, the phase-matching temperature of the PPMgLN in the laser cavity was investigated by changing the crystal temperature. As shown in Figure 4, the phasematching temperature of PPMgLN intracavity frequency doubling is $35.2^{\circ} \mathrm{C}$, and the output power is very sensitive to the crystal temperature. The temperature change of $1^{\circ} \mathrm{C}$ could cause nearly $100 \mathrm{~mW}$ output drop. In contrast, in the single-pass scheme, the phase-matching temperature is $38^{\circ} \mathrm{C}$, which is $2.8^{\circ} \mathrm{C}$ higher than that in the intracavity scheme.

To evaluate the reliability and stability of our green laser, continuous operation for 100 hours was carried out. During that period, no drop in green output power was observed, implying that the photorefractive damage is negligible in our experiments. As shown in Figure 5, the change of the green output power is less than $2.5 \%$ for 100 hours, indicating that the PPMgLN crystal is a practical material to use in generating stable green laser light. Longer-time experiment for evaluating the stability of the PPMgLN crystal is in process.

\section{Conclusions}

CW power of $908 \mathrm{~mW}$ at $532 \mathrm{~nm}$ with $33.5 \%$ optical-tooptical conversion efficiency has been obtained from a $0.5 \mathrm{~mm}$ thick, $10 \mathrm{~mm}$ long PPMgLN crystal in an intracavity frequency-doubling scheme. It has been shown that the efficiency of the intracavity scheme can be much higher than that of single-pass frequency-doubling scheme, indicating that we can obtain higher efficiency and power by employing the intracavity scheme. At the phase-matching temperature, the output power has shown stable operation for more than 100 hours, and the change of the output power is less than $3 \%$ at the different transverse positions of the PPMgLN. The experiment results clearly indicate that practically compact and highly efficient green lasers can be realized based on bulk PPMgLN crystals if the uniformity of the crystal is high. We

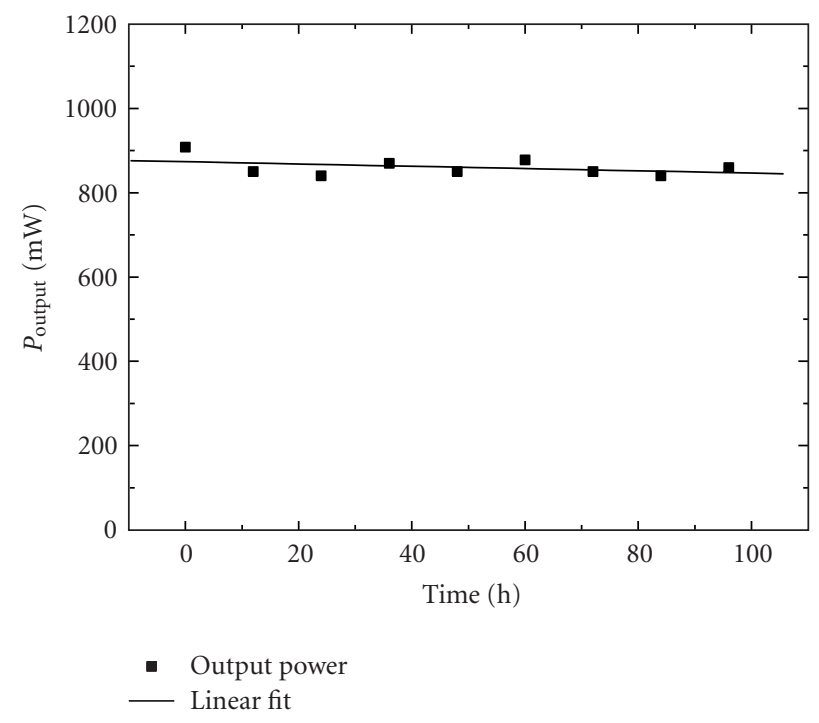

Figure 5: $532 \mathrm{~nm}$ output power versus work time for ISHG at phase-matching temperature.

expect that optical-to-optical conversion efficiency of more than $40 \%$ could be achieved if the AR coating of the PPMgLN crystal is improved.

\section{Acknowledgments}

The authors thank the C2C Link Corporation for helpful discussion as well as providing the high-quality PPMgLN nonlinear crystal. This work was supported by the Nation High-Tech R\&D Program ("863" Program, Contract no. 2006AA030103), the National Key Technologies R\&D Program (Contract no. 2006BAK12B13), and the National Knowledge Innovation Program (Contract no. KACX1-11).

\section{References}

[1] M. M. Fejer, G. A. Magel, D. H. Jundt, and R. L. Byer, "Quasi-phase-matched second harmonic generation: tuning and tolerances," IEEE Journal of Quantum Electronics, vol. 28, no. 11, pp. 2631-2654, 1992.

[2] G. D. Miller, R. G. Batchko, W. M. Tulloch, D. R. Weise, M. M. Fejer, and R. L. Byer, "42\%-efficient single-pass cw secondharmonic generation in periodically poled lithium niobate," Optics Letters, vol. 22, no. 24, pp. 1834-1836, 1997.

[3] S. Kurimura, N. E. Yu, Y. Nomura, M. Nakamura, K. Kitamura, and T. Sumiyoshi, "QPM wavelength converters based on stoichiometric lithium tantalate," in Advanced Solid-State Photonics (ASSP '05), vol. 98, pp. 92-96, Optical Society of America, Vienna, Austria, February 2005.

[4] N. Pavel, I. Shoji, T. Taira, et al., "Room-temperature, continuous-wave 1-W green power by single-pass frequency doubling in a bulk periodically poled $\mathrm{MgO}: \mathrm{LiNbO}_{3}$ crystal," Optics Letters, vol. 29, no. 8, pp. 830-832, 2004.

[5] S. V. Tovstonog, S. Kurimura, and K. Kitamura, "High power continuous-wave green light generation by quasiphase matching in Mg stoichiometric lithium tantalate," Applied Physics Letters, vol. 90, no. 5, Article ID 051115, 3 pages, 2007. 
[6] L. Y. Liu, M. Oka, W. Wiechmann, and S. Kubota, "Longitudinally diode-pumped continuous-wave 3.5-W green laser," Optics Letters, vol. 19, no. 3, pp. 189-191, 1994.

[7] V. Pruneri, J. Webjörn, P. St. J. Russell, J. R. M. Barr, and D. C. Hanna, "Intracavity second harmonic generation of $0.532 \mu \mathrm{m}$ in bulk periodically poled lithium niobate," Optics Communications, vol. 116, no. 1-3, pp. 159-162, 1995.

[8] K. S. Abedin, T. Tsuritani, M. Sato, and H. Ito, "Integrated intracavity quasi-phase-matched second harmonic generation based on periodically poled Nd:LiTaO 3 ," Applied Physics Letters, vol. 70, no. 1, pp. 10-12, 1997.

[9] M. Pierrou, F. Laurell, H. Karlsson, T. Kellner, C. Czeranowsky, and G. Huber, "Generation of $740 \mathrm{~mW}$ of blue light by intracavity frequency doubling with a first-order quasi-phasematched $\mathrm{KTiOPO}_{4}$ crystal," Optics Letters, vol. 24, no. 4, pp. 205-207, 1999. 

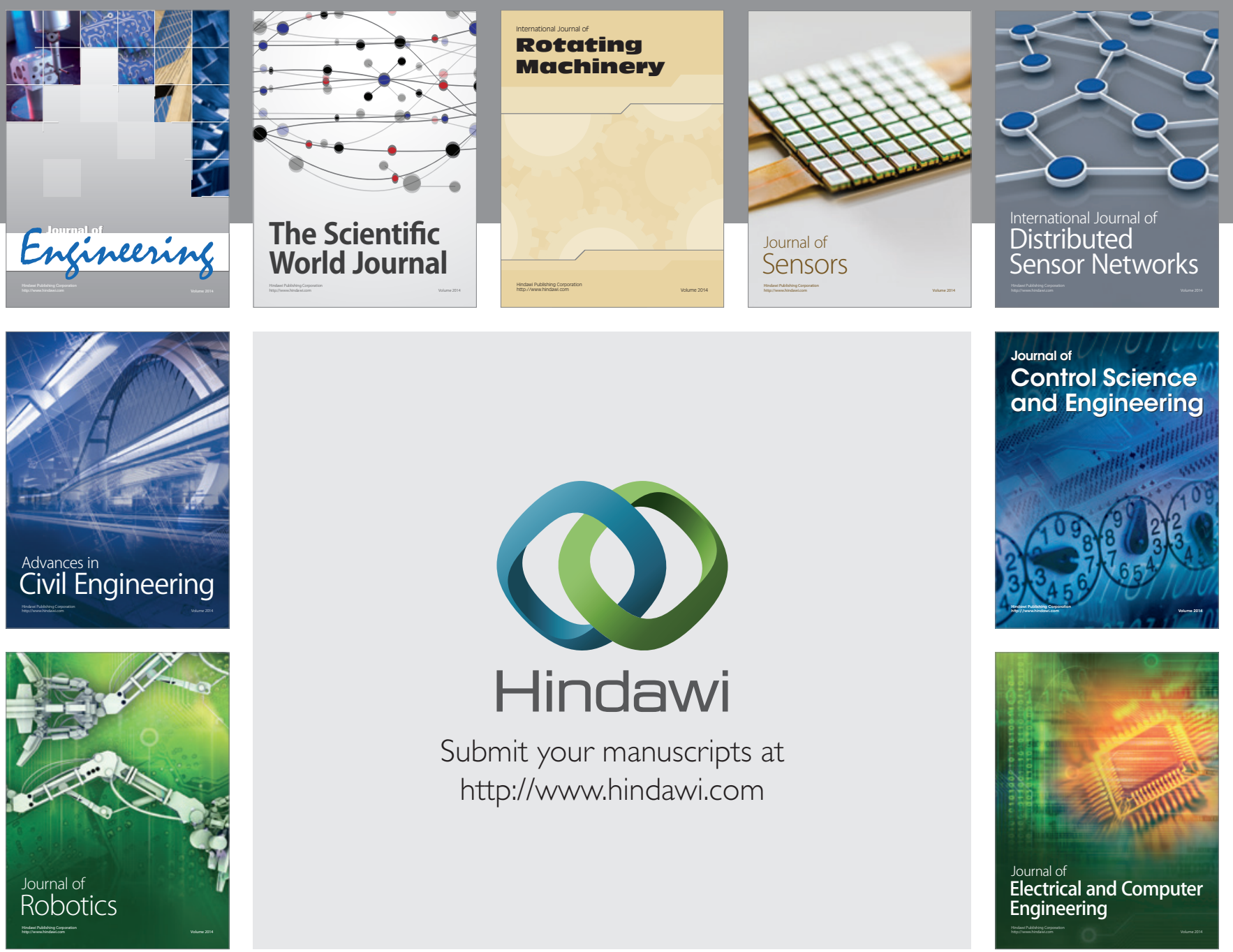

Submit your manuscripts at

http://www.hindawi.com
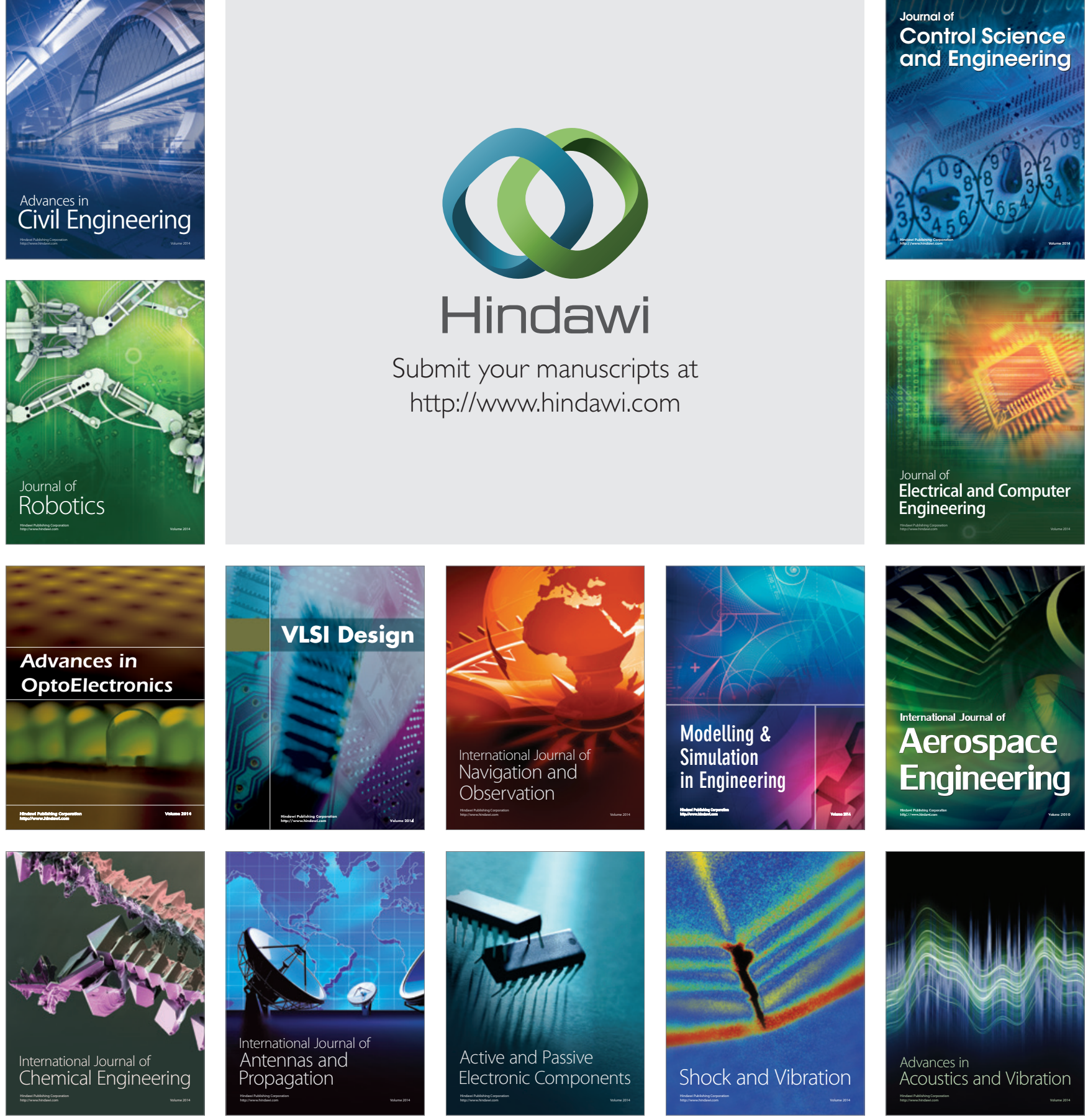